\title{
Geometric Optimization of Turbocharger Compressor and Its Influence on Engine Performance
}

\author{
Fangming Zhang and Roland Baar \\ Technical University of Berlin, Institute for Internal Combustion Engines, 10587 Berlin, Germany
}

\begin{abstract}
This paper consists of two parts: aerodynamic and mechanical multi-objective optimization for centrifugal compressor impeller through combining the three dimensional fluid dynamic simulation module CFX 16.1, the static structure in the ANSYS Workbench and the optimization software optiSLang; and a comparison and analysis of the effects of the optimized compressor on the engine performance by the one dimensional simulation tool GT-Power. In the process of optimization, the compressor design point is regarded as the optimizing point, while impeller blades and hub line were parameterized through the Bezier curve. Pressure ratio, isentropic efficiency, quality and maximum deformation and maximum internal stress of the impeller were defined as the output conditions. MOP module was then adopted in optiSLang for the parameters sensitivity analysis and mapping relationship modeling between the impeller parameters and the objective functions. The genetic algorithm is applied to find out and validate the optimal design. Through 1D simulation tool GT-Power, the influence of the optimized compressor on rotational speed of the turbocharger, backpressure and pumping loss under different engine operating conditions is analyzed and compared.
\end{abstract}

\section{Introduction}

Under the increasingly strict environmental pollution and emissions law, interest in the development of turbocharged engines has grown rapidly in recent year due to their low fuel consumption and high power characteristics. At the same time the research on the turbocharger is also changing with each passing day. Attempts to overcome the hysteresis phenomenon, problems of high knock rating, and high loss rate have become a great challenge to the development of new turbocharged engines. The design of the compressor impeller has a direct influence on key factors including the maximum working range, exhaust gas utilization, and service life, as well as exerting an important influence on the power and economy of the engine. With the rapid development of computational fluid dynamics software and the rapid expansion of optimization algorithms in engineering, the development cycle of the turbocharger has been greatly shortened, and the costs were reduced accordingly.

Multi-objective optimization problems are widespread in the theoretical research and engineering application and widely popularized, their computation complexity will also be increased with the increase of dimensions, the quantity of sub-objective shows exponential growth with the rise of relevance. Maintaining the low dimension and controlling the number of objectives will greatly reduce complexity and computation burden. Correlation and relative importance of each sub-objective will be embodied through the weight coefficient. Besides, the overall multi-objective optimization problem will also be analyzed and solved through the resulting comprehensive evaluation function. A series of optimal solution set existed in multi-dimensional space according to different design objective, arbitrary units of this solution set will make each sub-objective value to achieve the overall optimum, this solution set is called as Pareto solution set. In order to solve multi-objective optimization problems, a series of algorithms have been carried out. Geller and Schemmann [1] conducted simulation analysis of three dimensional flow field and statics calculation on centrifugal compressor impeller on ANSYS Workbench platform, and combined with the genetic algorithm of optiSLang optimization software to conduct optimization design and analysis, the results showed that under guaranteed equal efficiency, maximum stress of impeller was reduced by $40 \%$. Wei-guo Zhao et al. [2] combined the advantages of artificial neural network with the genetic algorithm, with the simulation results of CFD three-dimensional fluid as the optimal initial population, they carried out the optimization design of centrifugal pump impeller and overall simulation with redesigned volute, the efficiency at the design operating point improved by $1.59 \%$, non-operating points are increased by $6 \%$. But only through the three-dimensional aerodynamic optimization and ignored security such as blade mechanical strength limit, deformation, it will reduce the admissibility of some optimization results in the practical application. To this, Hildebrandt et al. [3] respectively conducted three dimensional flow field simulation, combined with three-dimensional fluid 
dynamics and simulation optimization of statics on centrifugal compressor, and verified through experimentation, the results showed that the optimal value difference was large, only the compressor performance obtained by the aerodynamic optimization of limiters obviously larger than the results obtained by two kinds of restriction of fluid optimal and security, and the working range was also greatly reduced. Dynaldo company [4] compared the Kriging approximation algorithm, support vector regression, artificial neural network and the MOP approximation (Metamodel of Optimal Prognosis) and evaluated their mapping effect of optimized variables and objective function, the results show that, the mapping effect established by MOP was not changed along with the increase of variable, while the mapping effect of other three kinds were less than $60 \%$ when the number of optimization variables more than 10 . Therefore, the MOP algorithm will be applied in this paper. The main objective of this paper is to establish, using ANSYS Workbench a three-dimensional fluid and static model for optimizing centrifugal-flow compressor, combined with MOP and genetic algorithm module in optiSLang, optimizing the geometric size of impeller. The influences of an optimized compressor on engine performance were finally analyzed and summarized by the 1D-simulation tool GT-Power.

\section{Optimization methods}

\subsection{Optimization object}

This paper will carry on the optimization to the compressor impeller with splitter blades. Due to the geometric limit of compressor casing, the diameter and the outlet height of the impeller are fixed, and the shroud curve on impeller meridian plane is maintained constant in the process of optimizing. Optimizing parameters are the angle, thickness on the meridional curve of blade and the shape of the hub control curve. The compressor impeller used in the experiment is shown in Figure 1.

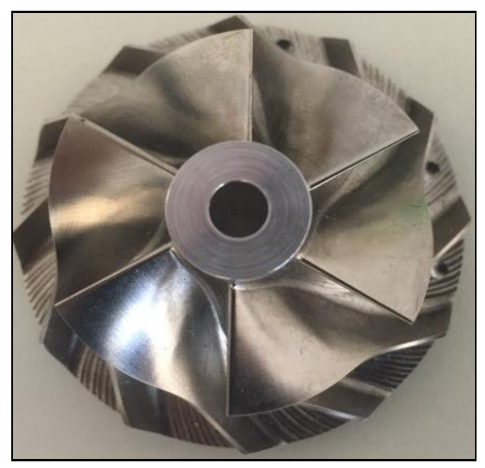

Figure 1. Impeller in the experiment

\subsection{Optimization objective}

The optimization of the compressor impeller is mainly carried out through two aspects of judging the operation safety and the work efficiency. Blade angle on leading edge and trailing edge, impeller clearance, blade slant
Angle, and blade shape trajectory and so on will directly determine the direction of motion and path of flow field into the cavity of the impeller, including the growth and decline of secondary flow, the size and distribution of the entropy production, the pressure gradient distribution on the blade surface, etc. The increase in blade thickness can increase the impeller's mechanical strength to a certain extent. But due to the increase of quality, fluid blocking rate causes the growth of rotational inertia and the decrease of effective inlet area. The contradiction between the optimization objectives, the nonlinear response characteristics have become the main objectives need to be solved in multi-objective problem, while the judgment of the final optimization results will be embodied with weights $w_{\mathrm{i}}$ according to the requirements.

$$
E_{\max }=\Sigma w_{\mathrm{i}} \cdot\left(T_{\mathrm{i}}-T_{\mathrm{io}}\right) / T_{\mathrm{i}}
$$

This paper will outline the optimization of the design point condition of compressor (speed: $140 \mathrm{kr} / \mathrm{min}$; flow: $0.08 \mathrm{~kg} / \mathrm{s}$ ). Optimization objective are detailed as the following objectives:

- Maximization of isentropic efficiency of whole turbocharging system

- Reduce the maximum stress lower than the allowable stress of impeller

- Reduce the maximum deformation and at least maintain original deformation

- Reduce the quality and at least maintain the original quality

- Increase and at least maintain the current maximum blocking flow

- Maintain or improve the supercharge ratio near surge area

\subsection{Optimization process}

The optimization process can be divided into three steps.

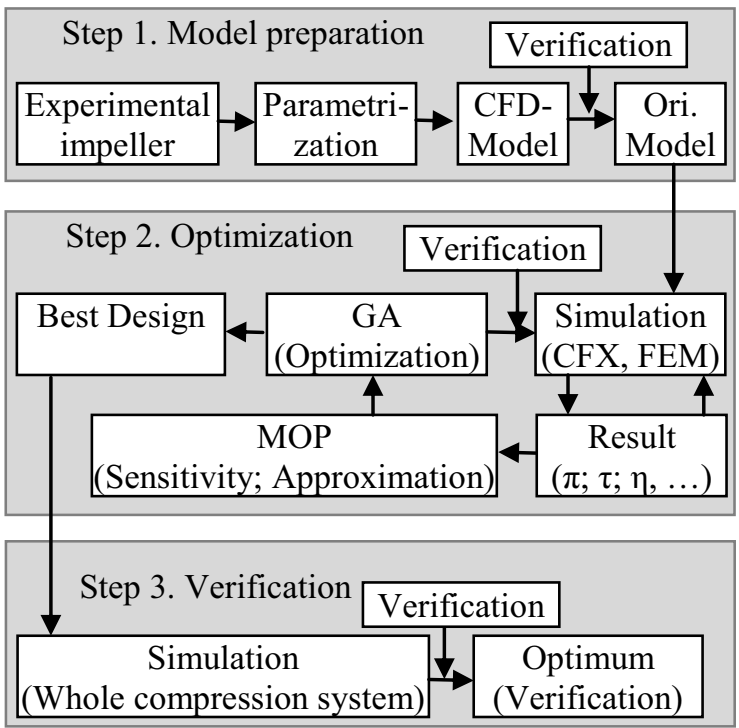

Figure 2. Optimization process

\subsubsection{Step 1: Model preparation}


For impeller optimization, following two premises must be met: first, the parametric modeling of impeller, by controlling parameters to establish different geometric features of the impeller model. Second, establish the same compressor system as the impeller in experiment, compared the experimental results through CFD and FEM simulation and ensure the correctness of the model. As a precondition for optimization calculation, blade parametric model will directly affect the optimal scale and complexity; also directly affect the accuracy of the optimization. In this paper, it will conduct recognition processing of structure angle and thickness on the original blade through the ANSYS Designmodeler 16.1 CAD-Import function, using Beta Angle and the thickness curve to describe blade type, all are adopted the 4-order Bezier curve fitting. Control points $P_{\mathrm{i}}$ of Bezier curve are uniformly distributed within the scope of blade design, each control point can be moved along the vertical or horizontal axis to determine the new blade model. The formal description of Angle and the thickness curve is given by:

$$
P(x)=\Sigma P_{\mathrm{i}} \cdot R_{\mathrm{i}, \mathrm{x}}(x) \quad x \in(0,1)
$$

where $R_{\mathrm{i}, \mathrm{x}}(x)$ are Bernstein basis polynomials of degree $\mathrm{i}$. The total of optimizing parameters is 47 , of which the main blades and splitter blades including angle and thickness are 20 parameters of each.

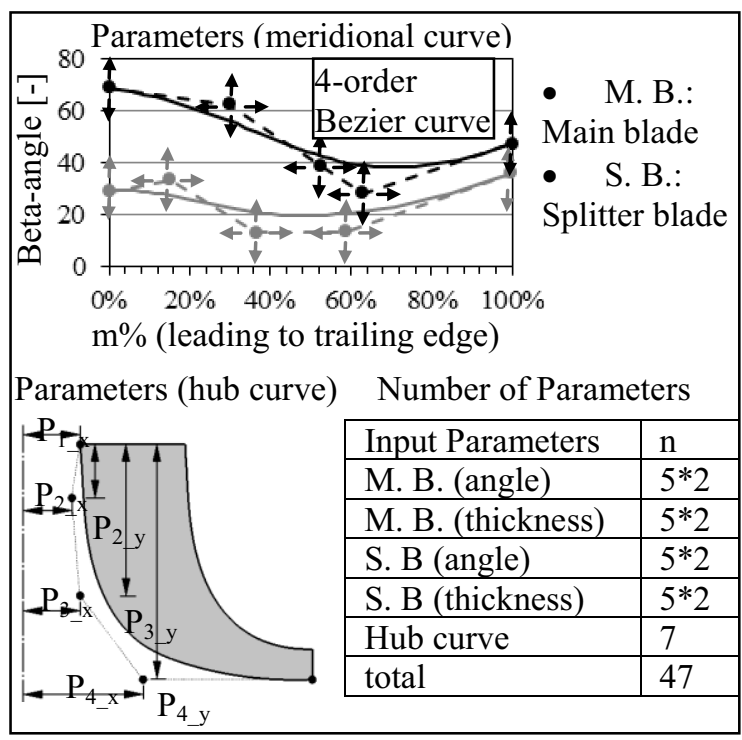

Figure 3. Parameters on meridional and hub curve

For hub curve, the parametric model is established with the aid of SolidWorks, ANSYS is imported in for identification. The maximum characteristic of Bezier curve is that it can use less control point to describe and generate complex and smooth curve. Its convex hull property can make it always fall in the convex hull of control points, so the basic line type is unable to have fierce volatility [5], and operation is simple, these characteristics are beneficial to the parametric design of space curve and narrow space dimension.

\subsubsection{Step 2: Optimization}

optiSLang provided rich optimization schemes for solving the problem of multi-objective optimal value [6], mainly are three categories: gradient method, natural inspiration method (including genetic algorithm, particle swarm optimization (PSO) algorithm, etc.) and adaptive method. Genetic algorithm (GA) is suitable for solving the optimization problem of geometric parameters of the impeller with its high efficiency, global superiority, multi-parameter optimization, etc.

In the genetic algorithm, a conduct fitness analysis on new individual of each generation after restructuring is required, and variation, if 3-D flow field and mechanics analysis were carried on the individuals one by one in each generation, it is bound to increase operation difficulty and simulation time. By using MOP module in optiSLang, it will not only precisely establish the mapping relationship of optimization parameter and objective function, conduct fitness analysis on new individual by establishing approximation model, and nonlinear sensitivity analysis on optimization of parameters, so as to eliminate the interference of nonsensitive parameters on the approximation model, and improve the quality of optimization. While the initial population individuals shall be conducted 3-D flow field and statics calculation one by one, physical results such as the isentropic efficiency, compression ratio, and maximum stress are provided to the MOP as raw data. optiSLang software provides the CoP (Coefficient of Prognosis) parameters to judge the quality of the established approximation model, when the value is close to $100 \%$, it indicates the mapping relationship is better. For nonlinear output, of course, the convergence results of the input will reduce the $\mathrm{CoP}$ value, which may cause the results obtained by approximation model deviate from the actual values, thus influence optimization results. optiPlug is respectively connected to ANSYS and optiSLang, convenient for the exchange, edit of input, output data under these two conditions. In addition, this paper adopts the optiSLang integrated Latin Hypercube method in optiSLang for random sampling of the overall design space. Compared with the Monte Carlo random sampling method, this method has advantages of more uniform coverage of the individual in the whole $n$ dimensional space, number of samples are 10 times less than Monte Carlo method, etc. [7].

\subsubsection{Step 3. Best design validation}

Ultimately, verification of the optimal blade design will be performed. Based on the optimized model, the working performance of the blade at each operation point is calculated and analyzed.

\subsection{D simulation model}

In order to improve the precision of the model, and shorten the simulation time, first of all, it conducts 3-D modeling on the whole turbocharging system, this model is determined as original model through steps such as non-dependence analysis of gridding and contrast experimental data. Secondly, a three-dimensional single 
passage optimization model is established based on the original model, with ANSYS Workbench as work platform for steady simulation and statics calculation of integral impeller. Finally, optimization is carried on through optimizing software optiSLang, and the optimal impeller model is substituted into the whole model, and then its simulation results are compared with original model. In Figure 4, the left picture is the 3-D whole compression model, and right picture is single passage impeller model.

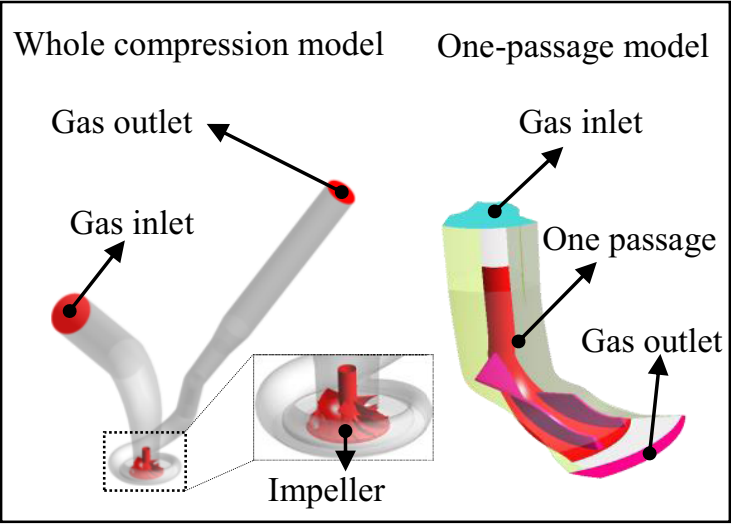

Figure 4. Whole turbocharging model; single passage model

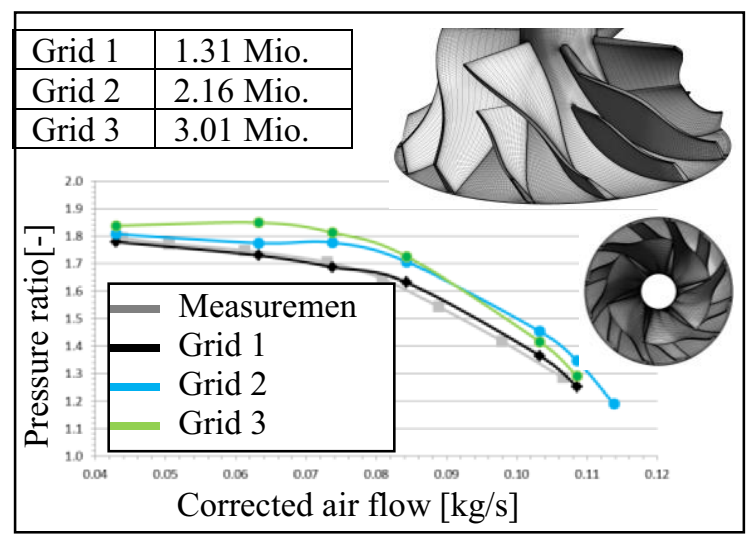

Figure 5. Compressor map under different grids

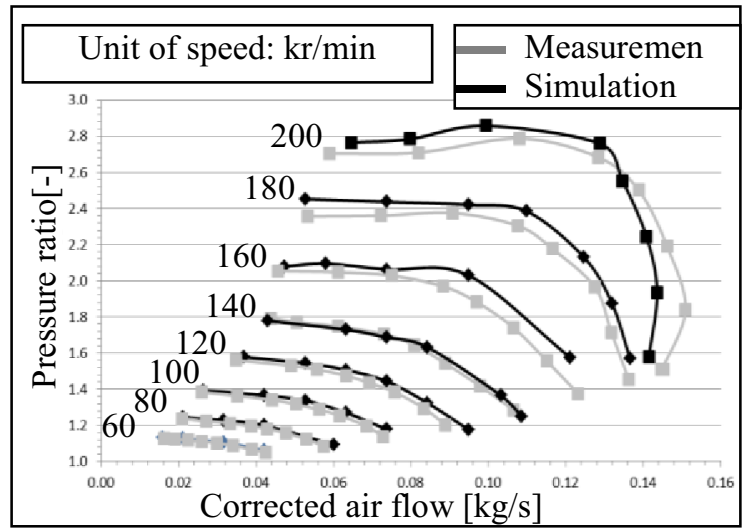

Figure 6. Comparison simulation results with measurement

Impeller passage grid is automatically generated by Turbogrid module. Turbulence model is chosen as the SST model, a more accurate description of near wall features and calculation of far field flow condition are advantageous to the accurate calculation of aerodynamic loss. Based on the finite volume method to solve the 3-D Reynolds average Navier - Stokes equation, second order center difference method is adopted to conduct space discrete, the viscous shear mode of Total Energy is added for precise calculation of the flow loss. The frozen rotor method is used to realize the combination of sensory transfer and localized flow field. The system boundary is set as adiabatic with no slip. The blade wall surface in flow field is dispersed by O-grid, inlet, outlet and the clearance of volute and flange are meshed by using $\mathrm{H}$ grid. It can be known from research on the impeller nondependence grid that, as shown in figure 5, the simulation values in grid 2 and 3 were larger from experimental data, simulation results obtained through grid 1 were much better with the regional Y-plus values at less than 3, thus conforming to the requirements of the SST turbulence model. The working condition curve under each speed obtained by the first grid from the simulation was close to the experimental value, as shown in figure 6 , and will be adopted.

\section{Analysis of optimization results}

\subsection{Sensitivity analysis}

The whole process of impeller geometry optimization adopted eight core CPU simulation, simulation time were 15 days, the original species are selected as 200 , with a total of 20 simulation failures $(<15 \%)$, after 90 generations of genetic variation, mutation rate is $20 \%$, finally the optimization results obtained.

Figure 7 shows the MOP model of output parameters. Apart from to the pressure ratio, CoP value of other output parameters are higher than or equal to $90 \%$, this indicates that the mapping relationship of optimization parameter and objective function is better. It can be known from impeller maximum stress, maximum deformation MOP model that the output values are mainly affected by the eight parameters, of which parameter FD1207 had the greatest influence, namely the geometric angle on top of main blade leading edge. The effect on isentropic efficiency is more than $60 \%$. The influence of optimization parameters on the pressure ratio and quality is relatively uniform. The impeller quality is mainly affected by six control parameters of hub curves. Pressure increase caused by rotation of the impeller is mainly affected by the angle on the bottom of blade trailing edge.

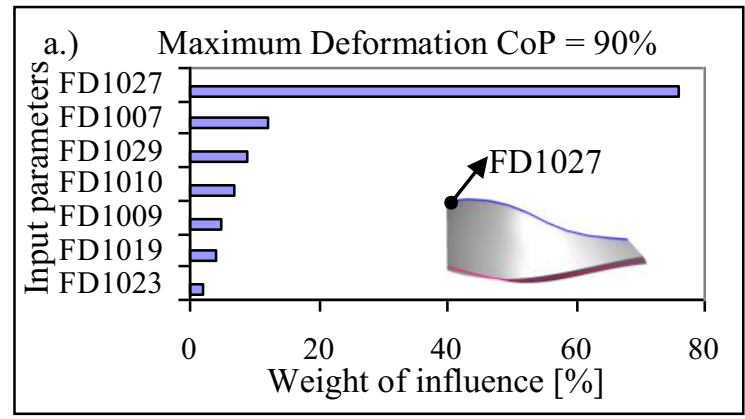



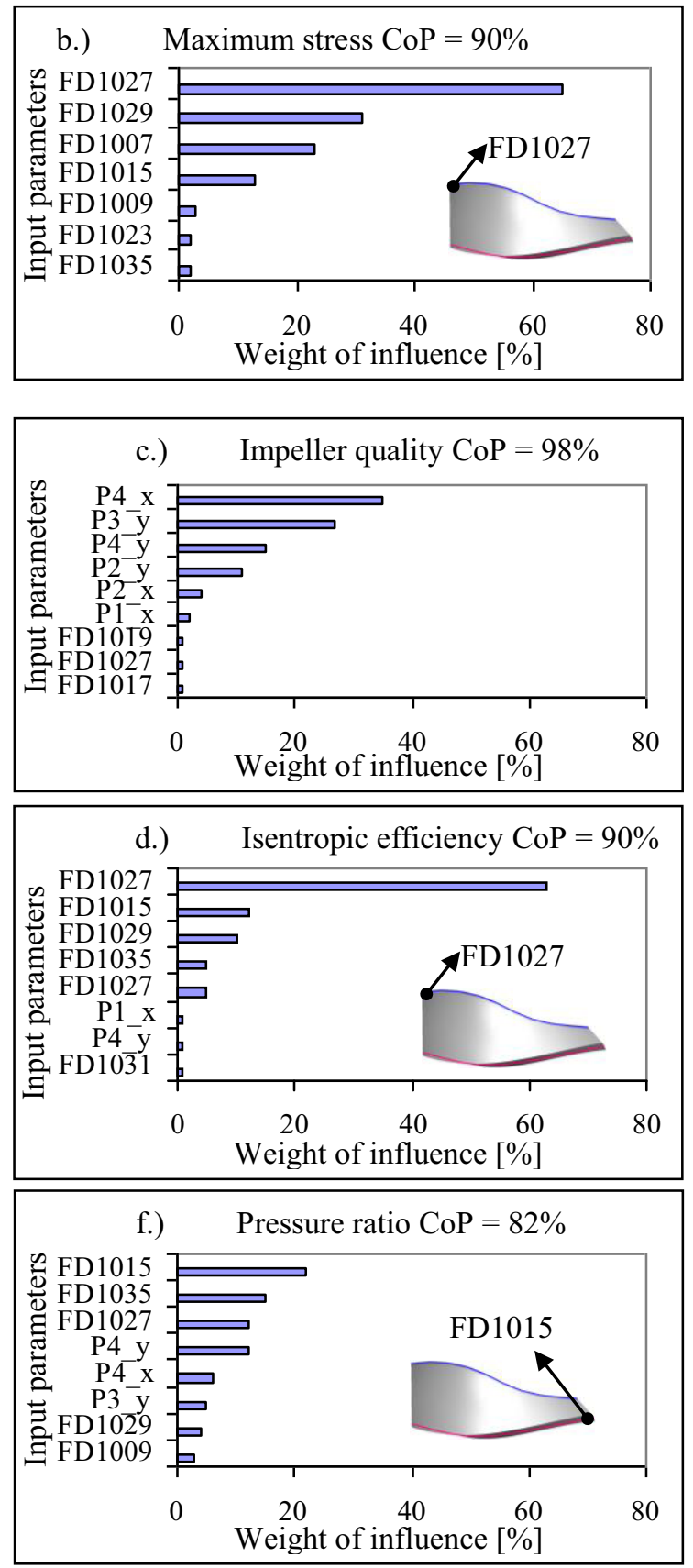

Figure 7. Result of sensitivity analyses

\subsection{Optimization analysis and verification}

In the process of global optimization, MOP model has created a total of 9000 design points, as shown on the bottom half of figure 8 , pressure ratio is converged by generations, and close to the maximum.

Red marked points are ultimate Pareto solution sets. As a multi-objective optimization problem, the optimal solution for pressure ratio will be subject to other optimization objectives. Therefore, Pareto solution is not the maximum value of compression ratio. Similarly, the isentropic efficiency is also converged by generations and close to the maximum value.

As shown in Fig. 9, X-coordinate is the isentropic efficiency, $\mathrm{Y}$-coordinate is the pressure ratio, Pareto solution set has a total of 1047 design points, focused on the right position of figure, and is superior to the obtained isentropic efficiency of original model and pressure ratio benchmark. Considering other optimization objectives, the efficiency of some optimal solutions is slightly lower than the highest value.

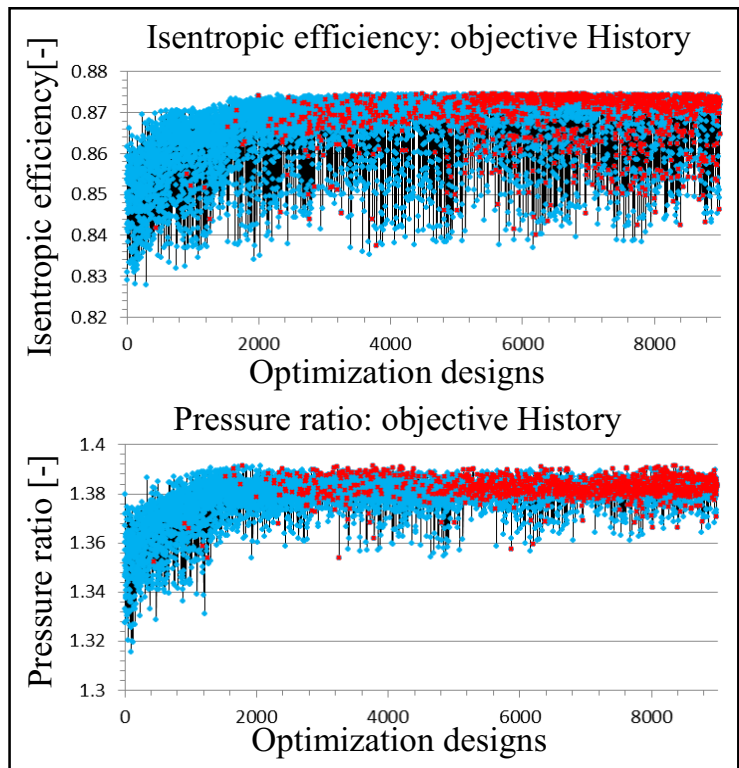

Figure 8. Efficiency and pressure ratio history

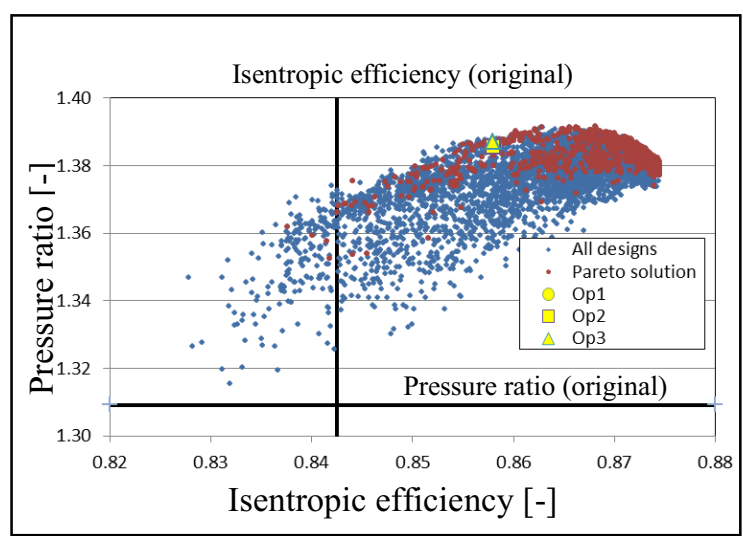

Figure 9. Optimal design in 'MOP' model

In the Pareto solution set, the optimal value will be found out by solving the equation 1 with the weight coefficient of all objectives. In view of the impeller job security and efficiency, the isentropic efficiency and the maximum stress are the most important optimization objectives.

Table 1. Comparison of three optimized compressors

\begin{tabular}{|c|c|c|c|}
\hline & Op1 & Op2 & Op3 \\
\hline Pressure ratio & $6.11 \%$ & $5.88 \%$ & $5.65 \%$ \\
\hline $\begin{array}{c}\text { Isentropic } \\
\text { efficiency }\end{array}$ & $1.31 \%$ & $1.66 \%$ & $1.66 \%$ \\
\hline $\begin{array}{c}\text { Impeller } \\
\text { quality }\end{array}$ & $-7.42 \%$ & $-7.42 \%$ & $-5.65 \%$ \\
\hline $\begin{array}{c}\text { Maximal } \\
\text { miser stress }\end{array}$ & $-1.68 \%$ & $-3.74 \%$ & $-5.52 \%$ \\
\hline $\begin{array}{c}\text { Maximal } \\
\text { deformation }\end{array}$ & $-3.87 \%$ & $-0.26 \%$ & $-0.26 \%$ \\
\hline
\end{tabular}


By the method of given weights on the objective functions, rating on the 1047 design points has been conducted. As a result, the rating of Op1, Op2 and Op3 design point is the highest. Therefore these three design point will be regarded as the final design points.

Table 1 gives the comparison of the three kinds of optimization results with the original model. A negative value indicates decrease. Research shows that the maximum stress, deformation and quality are decreased, the maximum loss is around $7 \%$, and pressure ratio and isentropic efficiency also increase accordingly. In order to verify the accuracy of approximation model, 3-D single flow passage simulation and mechanical calculation on Op1, Op2 and Op3 has been conducted again. Figure 10 shows the comparison of verification results with approximate model results, the results are conform to expectation. Under the design point conditions, the maximum difference of pressure ratio for Op1, Op2 and Op 3 obtained by the approximation model and the results of verification simulation is about 0.003 . Besides, the isentropic efficiency has a decrease of about 0.005 , and the verification result is close to the approximation result, so the accuracy of the approximation model is higher.

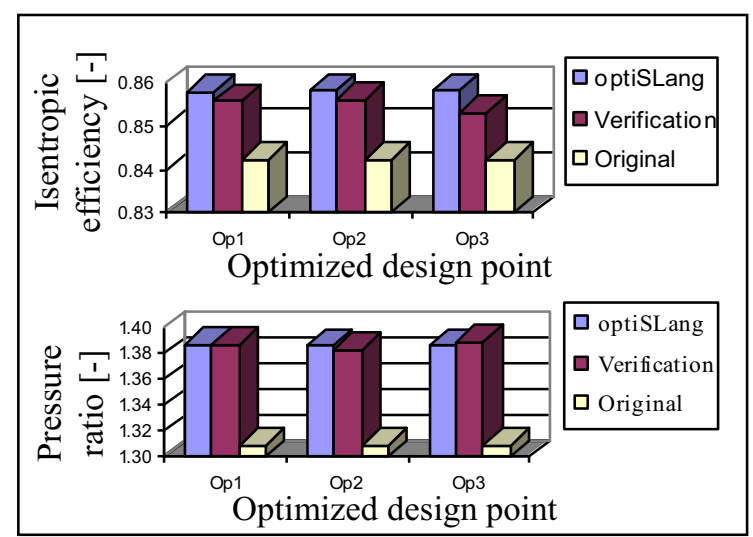

Figure 10. Comparison of verification results with approximation model result

\subsection{Results comparison}

Finally there is a whole compression system simulation with different mass flow in the speed of design point and comparing the simulation result of optimal impeller design and original model. As seen in Fig. 11, under the design speed, the isentropic efficiency of optimal impeller at design point has slight increase $2.24 \%$ and the increase of pressure ratio is $4.34 \%$; for mass flow less than $0.083 \mathrm{~kg} / \mathrm{s}$ (mass flow at design point) the optimized compressor maintains fast the same efficiency compared to the original model; when the optimization model has a large mass flow (greater than $0.083 \mathrm{~kg} / \mathrm{s}$ ), the increases of efficiency and pressure ratio are greater; the working range of the optimized compressor under design speed is obviously increased and the maximum bypassed flow into the impeller (choke flow) is $4.85 \%$ increase compared with original model. Known from the comparison of the maximum stress and the maximum deformation of the impeller (shown as Fig. 12), the maximum stress on the blade after optimization is reduced by $1.33 \%$ and the maximum deformation is reduced by $2.52 \%$. In general, the optimization result conforms to the prediction. Due to the consideration of security, the growth of aerodynamic optimization is relatively small.

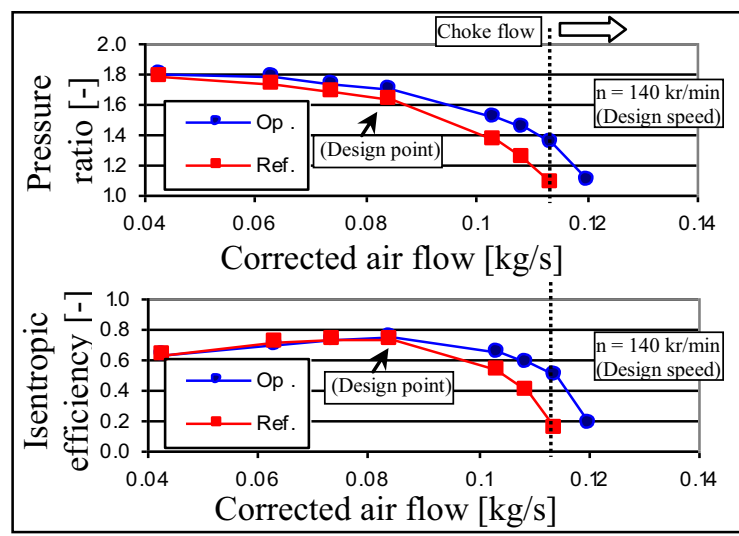

Figure 11. Comparison of efficiency and pressure ratio between optimized and original compressor

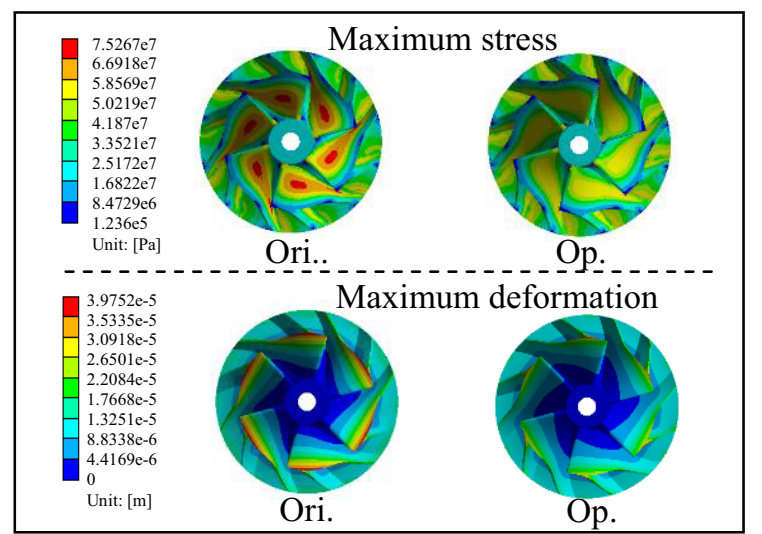

Figure 12. Comparison of maximum stress and deformation between optimized and original compressor

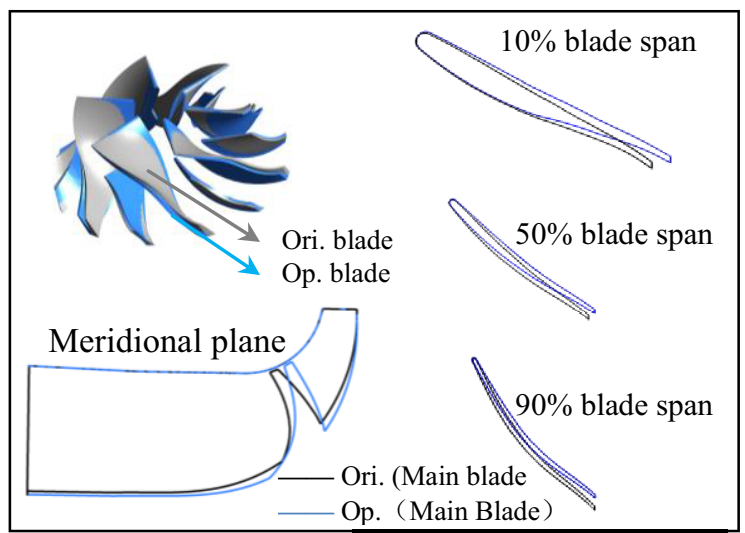

Figure 13. Comparison of blade profile between optimized and original compressor

The comparison of blade profile between original and optimized model is shown in Fig. 13: under design speed, the blade angle on the leading edge of optimized full blade is reduced, in order to meet the flow inlet angle and reduce the incident loss; after optimization the bottom of the trailing edge protrudes slightly backwards compared to the tip of trailing edge and the tile angle becomes small, which is results in increased pressure as well as isentropic efficiency (This conclusion is supported by sensitivity 
analysis). The middle of the optimized full blade is slightly inclined forward, so it can avoid low momentum fluids entering into the blade clearance as a result of centrifugal force to affect the increase of pressure. The excessive oblique of the trailing edge can not only affect the mechanical strength and it also leads to a decrease of the effective working area and an increase of force component for fluid in the passage, which is perpendicular to the blade; the tile angle of the optimized splitter blade is greater compared to the original model.

\section{1D analysis of influence of optimized compressor on motor performance}

\subsection{Comparison of compressor operating map}

Finally, the characteristics under each working conditions of the whole compression system are calculated. Figure 14 is the comparison of isentropic efficiency between original compressor and three optimized compressors. It can be seen that when the compressor produces pressure ration less than 1.8 , the isentropic efficiency of all the three optimized compressors' are significantly higher than the that of the original compressor and the highest isentropic efficiency respectively is increased by $0.34 \%, 0.29 \%$ and $0.2 \%$. However, when the operating point is located above the blue line, the original compressor shows better compression effect.

\subsection{D simulation model und comparison of results}

To analyze the influence of the optimized compressor on engine performance and quantify the improvement resulting from the optimization of compressor, this article will conduct experimental measurement of the engine, with which the original turbocharger is fitted as well as a sequent one dimensional simulation.

Figure 15 modularly demonstrates the GT model of turbocharged engine. The simulated object is a fourstroke 2L-diesel engine with four cylinders. In this model, the experimental data replaces combustion model and the pressure curve caused by inter combustion in cylinder, which are determined by engine speed and torque. Injection timing and its duration, the trajectories of the intake valve and exhaust valve are modeled by experimental data. Boost Pressure, speed of the compressor, high- and low-pressure exhaust gas recirculation rates and exhaust gas flow are adjusted by control system, to make the adjustment value reach the target. This paper shows steady simulation of the engine under full load and transient simulation under acceleration and low load, and took the original engine's performance as a benchmark, making the degree of optimization of back pressure in the engine, compressor speed and pumping loss under different conditions as the research object. Figure 16 shows the influence of optimized compressor on its rotational speed under full load condition. The vertical coordinate illustrates the ratio of speed reduction after optimization to the original model. It can be clearly seen that except for the fourth and fifth operating points, the speed of Op1 is lower than that of original compressor.

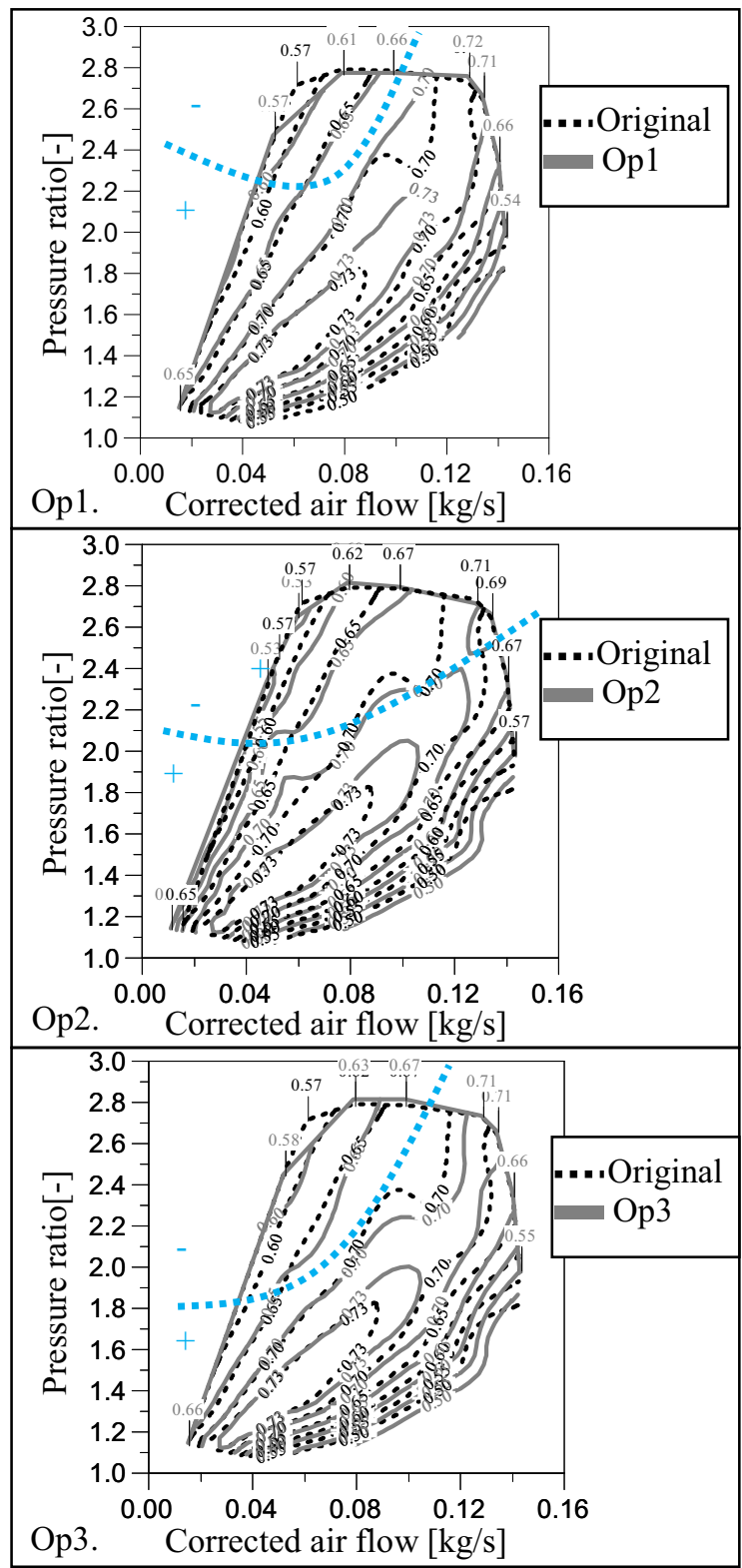

Figure 14. Comparison of isentropic efficiency between original compressor and three optimized compressors.

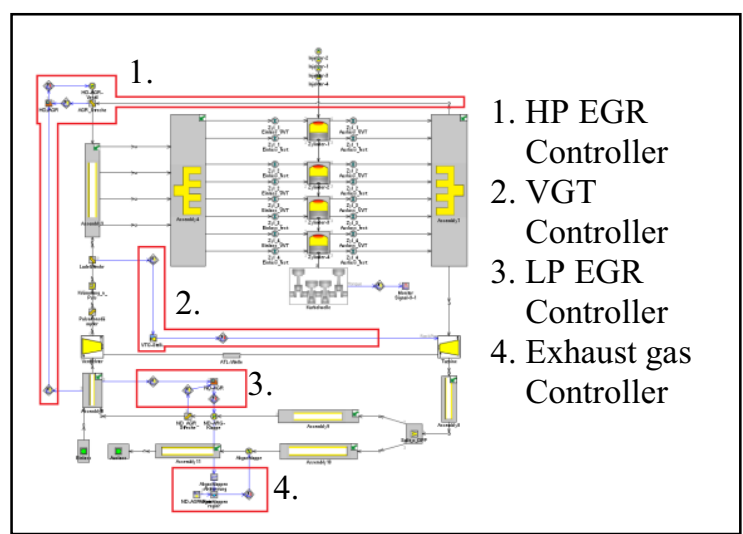

Figure 15. Modular GT-Model of turbocharged engine 


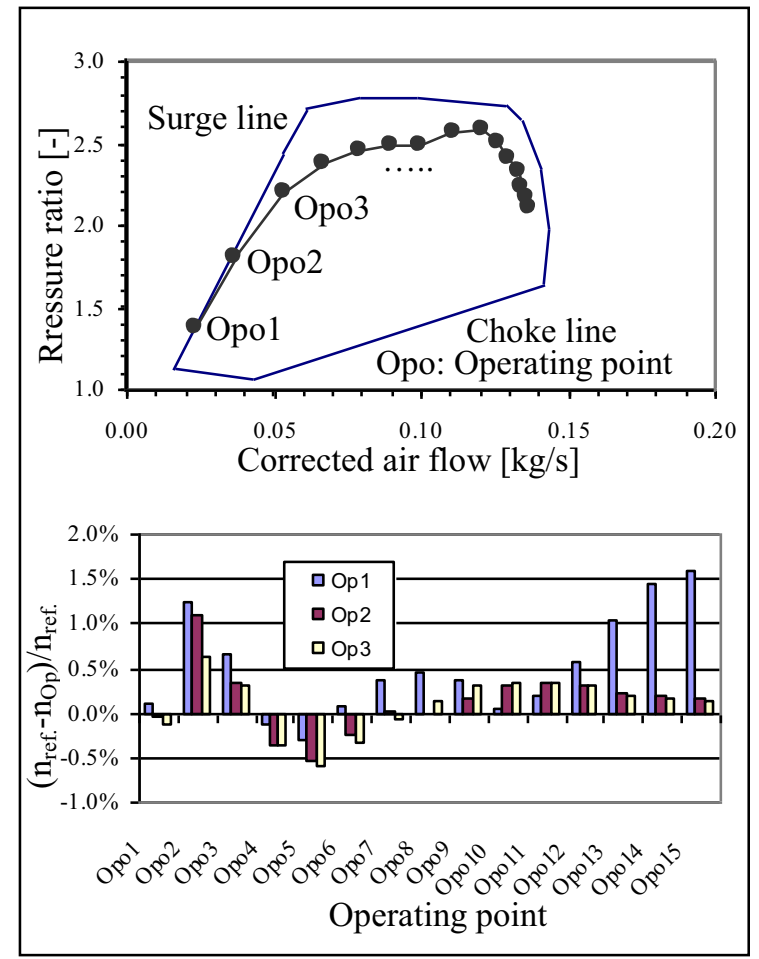

Figure 16. Speed of optimized compressor under full load condition

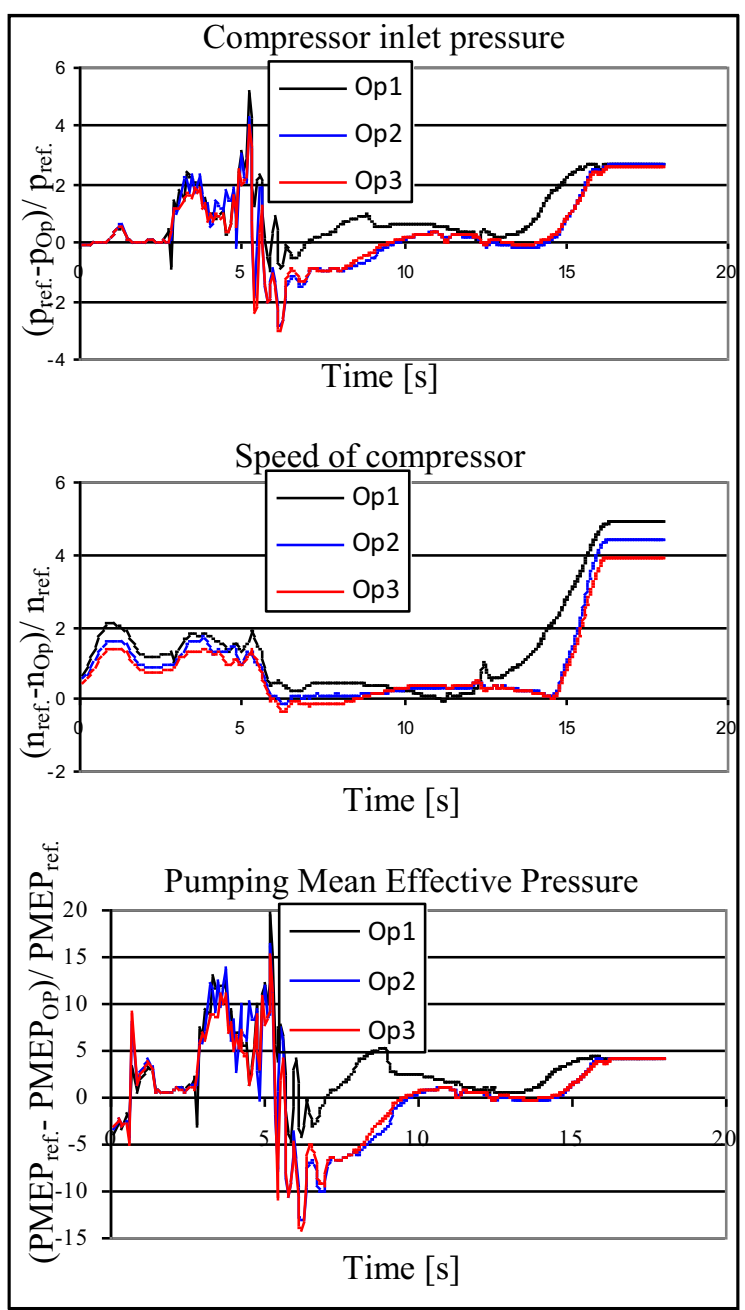

Figure 17. Comparison of results in acceleration condition
The increase of isentropic efficiency at these operation points also results in a reduction in the back pressure as well as in the load on the turbine blade. Figure 17 and 18 show the effect of three optimized compressors on engine performance in terms of acceleration and reaction under low load conditions. It can be seen that Op1 has a higher degree of optimization in the back pressure, compressor speed and the pumping loss.

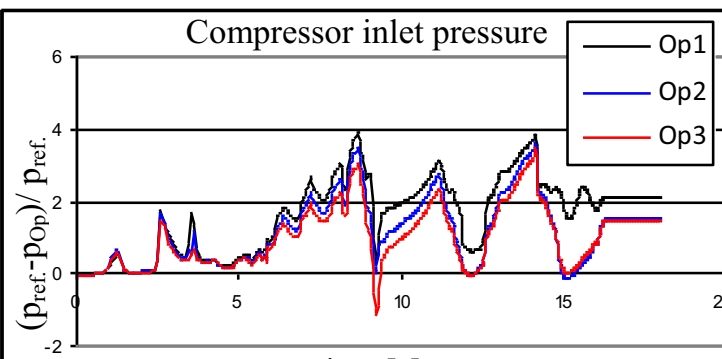

Time $[\mathrm{s}]$
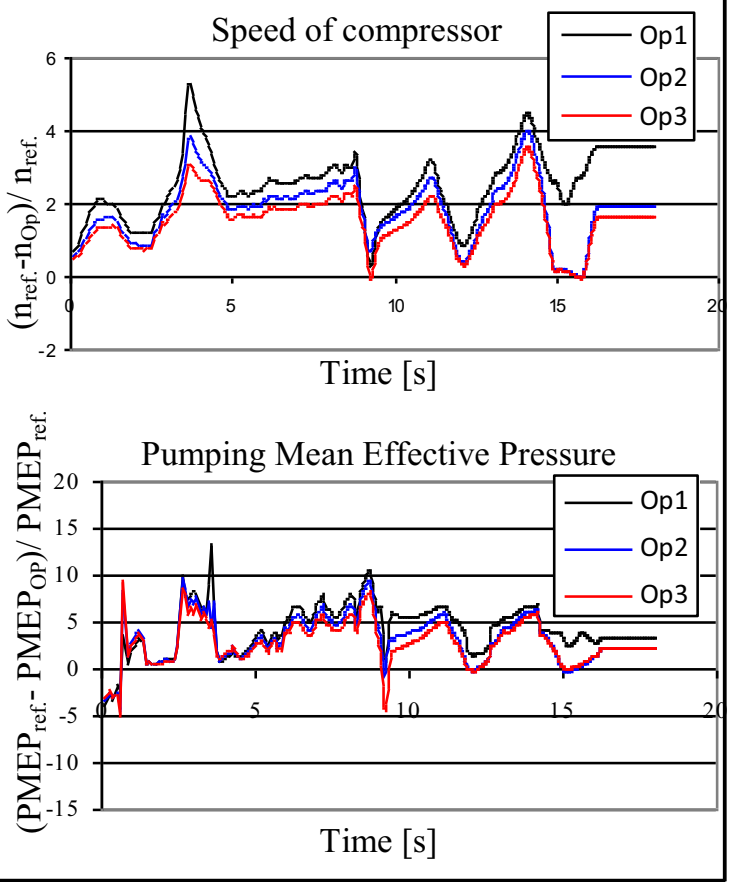

Figure 18. Comparison of results in low load condition

\section{Conclusions}

The multi-objective geometry optimization on compressor impeller was carried out through the coupling of CFD-simulation with Optimization method. The optimization process is as follows: conduct parametric modeling on impeller blade shape and hub curve by Bezier curve successful first, and verify the accuracy of three-dimensional CFD model, then using each module function in optimization software optiSLang. Using the Latin Hypercube method in optiSLang to establish the simulation sample set permits the advantages of less sample numbers and a more representative sampling. Through the sensitivity analysis of the results in aerodynamic and mechanics, the mapping relationship between the optimized parameters and the optimization objectives are established and the approximate model is obtained. Using the genetic algorithm in optiSLang to 
optimize and verify the model, finally the optimization model is compared with the original model, and an analysis and summary are carried out. The whole optimization system based on ANSYS and optiSLang coupling has features of simple operation, high efficiency, etc.

The aerodynamic performance and security of the optimized model were greatly improved. The optimization degree of isentropic efficiency and compression ratio was affected by different operating conditions. The maximum stress and maximum deformation of the impeller were also under control. A lower quality impeller will undoubtedly reduce its rotational inertia, and contribute to reducing the hysteresis phenomenon. Under the same condition of operation, the required speed of the optimized compressor is decrease. The pumping loss is reduced and the engine efficiency is increased. The optimized model has an overall smaller isentropic efficiency under high pressure ratio conditions.

\section{References}

1. M. Geller, C. Schemmann. Geometrieoptimierung eines Radialkompressorlaufrades mit Hilfe automatisierter Optimierungsmethode. 8. Weimarer Optimierungs- und Stochastiktage. 6 (2011)

2. W. Zhao, J. Sheng, J. Yang, J. Song. Optimization design and experiment of centrifugal pump based on CFD. Transactions of the Chinese Society of Agricultural Engineering (Transactions of the CSAE). 6 (2015)

3. Th. Hildebrandt, 1. Gresser, M. Sievert. Kennfeldverbreiterung eines Radialverdichters fuer Abgasturbolader durch multidisziplinaere CFDFEM-Optimierung mit FINETM/Turbo. 16.Aufladetechnische Konferenz. (2011)

4. Dynardo $\mathrm{GmbH}$. Methods for multi-disciplinary optimization and robustness analysis. Weimar. February 21 (2016)

5. B. Liu, Q. Yang, Y. Cao, P. Zhang. Optimization Design of a Centrifugal Compressor with Splitters. Journar of propulsion technology. 35. 7 (2014)

6. J. Will. OptiSLang inside ANSYS Workbench. Confidence by Design. Houston. June 20th (2012)

7. Dynamic Software and Engineering $\mathrm{GmbH}$. Sensitivty Analysis. Optimization and Robust Design with optiSLang and ANSYS Workbench 12.Tutorial 